
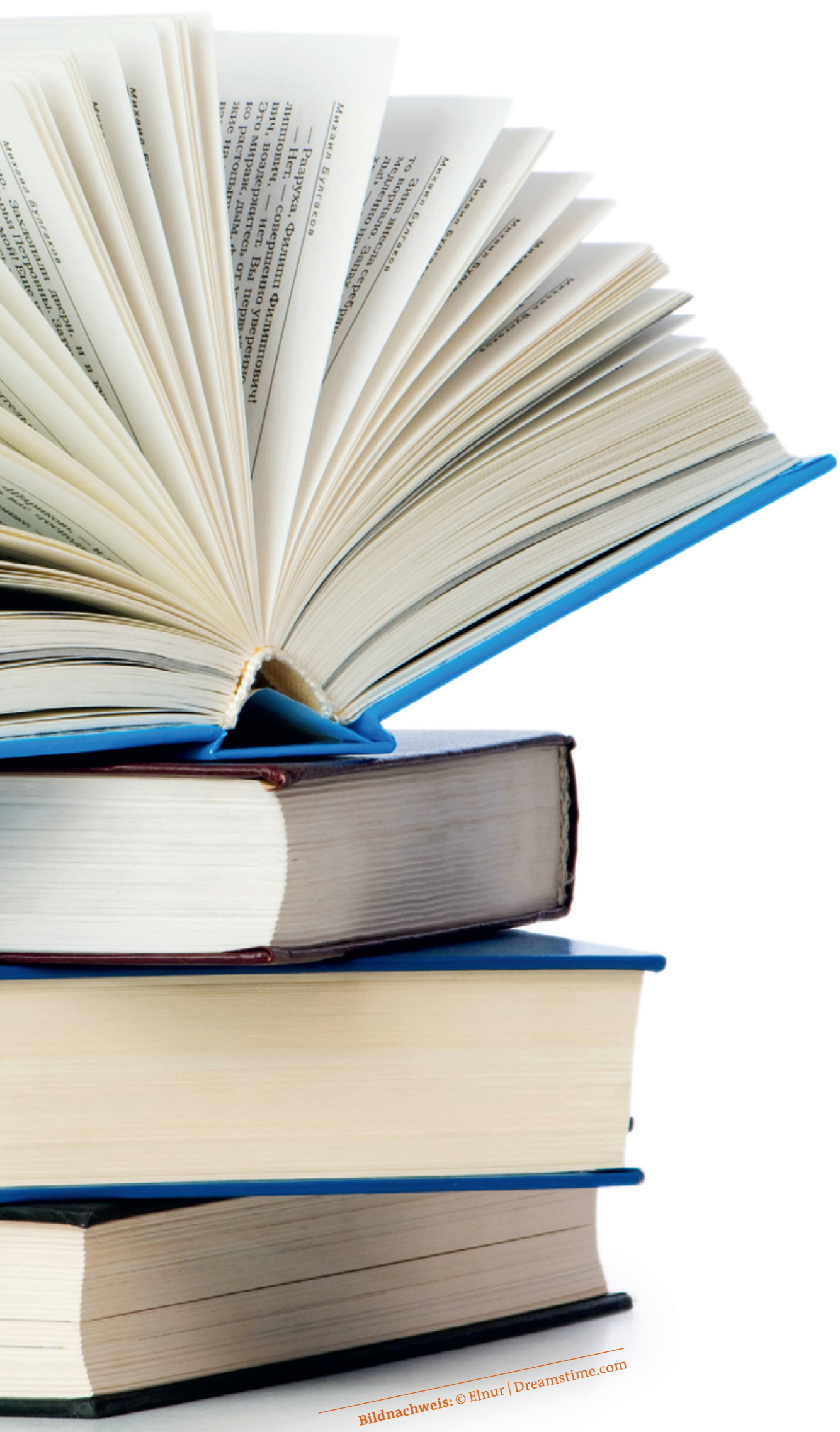

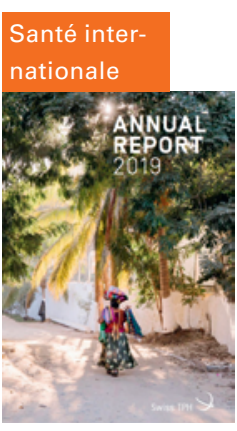

\section{Annual Report 2019}

Swiss Tropical and

Public Health Institute

Basel: Gremper AG,

2020

En tant qu'«alumnus» (ancien élève) de 1967 de l'Institut Tropical Suisse de Bâle, je reçois les rapports du désormais Swiss TPH et j'ai lu son Annual Report 2019.

Les rapports annuels ne sont pas forcément des lectures très stimulantes, mais celui-ci l'est: cahier attrayant, contenant des résumés et de multiples illustrations. Les départements scientifiques couvrent l'épidémiologie et la santé publique, les affections parasitaires et infectieuses, la formation académique, l'attention médicale dont la médecine des voyages, le consulting. Parmi la douzaine de domaines clés d'activité, dans 129 pays et avec une équipe comprenant 80 nationalités, j'ai relevé la recherche (de base, préclinique et clinique), le modelling statistique, le thème "Société, culture et santé», la santé sexuelle et reproductive, migration et santé, les systèmes et la politique de la santé.

Le dossier présente trois articles sur des thèmes spécifiques: "Le changement climatique est l'une des menaces les plus graves pour la santé globale»; "La résistance antimicrobienne va en croissant»: "Rétrospective: de l'Institut Tropical Suisse au Swiss TPH».

Dans la préface, le directeur, le Prof. Jürg Utzinger, souligne que toutes les activités vont dans le sens de l'Agenda 2030 pour le développement durable des Nations unies; un graphique parlant illustre les contributions aux 17 objectifs de cette stratégie.

Il s'agit d'une lecture nourrissante sur les objectifs, les réalisations et les défis que veut relever une institution phare dans son domaine, bien au-delà des frontières suisses. Elle retiendra l'attention de ceux qui s'intéressent aux diverses dimensions de la santé internationale, dont l'importance a été dramatiquement illustrée depuis ce printemps par la pandémie de Covid-19.

Dr méd. Jean Martin membre de la rédaction

jean.martin[at]saez.ch 


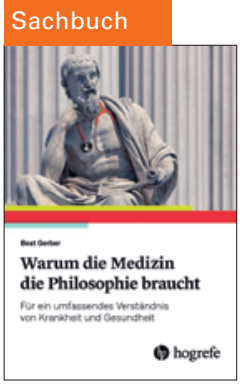

Warum die Medizin
die Philosophie
braucht
Beat Gerber

Bern: Hogrefe 2019

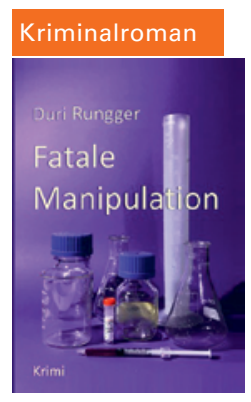

\section{Fatale Manipulation Duri Rungger}

Aarau: neobooks

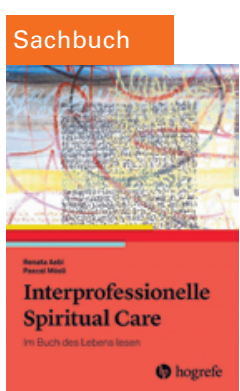

Interprofessionelle Spiritual Care

Renata Aebi,

Pascal Mösli

Bern: Hogrefe 2020
In letzter Zeit mehren sich die Anzeichen, dass die einseitige Ausrichtung der Medizin auf Naturwissenschaften - will sagen auf Biochemie und Molekularbiologie - die dringend nötige Korrektur zu erfahren beginnt. Ein Zeichen dafür stellt das Buch "Warum die Medizin die Philosophie braucht» dar. Der Autor, Beat Gerber, war jahrzehntelang praktizierender Allgemeininternist. Im Laufe seiner beruflichen Tätigkeit begann er sich intensiv mit Philosophie und philosophischen Fragestellungen in der Medizin auseinanderzusetzen. Sein Buch ist von einem Mediziner für Mediziner in einer gut verständlichen Sprache verfasst. Dies erleichtert den Einstieg in eine Welt, die spannender nicht sein könnte.

Gerber bespricht medizin-relevante Themen wie: Wann ist im ärztlichen Alltag Nichtstun besser als (überbordender) Aktivismus? Was bedeutet genau besehen "Nichtstun"? Welche Ziele verfolgen wir Ärzte und Ärztinnen bei unserer Tätigkeit? Krankheiten heilen, die Gesundheit der Patienten wiederherstellen. Nur: Was ist Krankheit und wie genau ist Gesundheit zu verstehen? Welche Rolle soll und darf Mitleid im ärztlichen Alltag spielen? Worin unterscheidet sich Mitleid von Empathie? u.a.m. Dabei geht es dem Autor erstens darum, aufzuzeigen, wie wir Ärztinnen und Ärzte an allen Ecken und Enden mit genuin philosophischen Fragestellungen konfrontiert sind. Zweitens möchte er in uns Ärzten und Ärztinnen eine genuin philosophische Geisteshaltung, eine Geisteshaltung des «kritischen Hinterfragens von Gewissheiten, vertieften Reflektierens über Bekanntes und Unbekanntes und Offenheit für Anderes und Neues» wachrufen. Da bleibt nur, Beat Gerber «viel Erfolg» zu wünschen!

\section{Dr. med., Dr. phil. Piet van Spijk Medicum Wesemlin}

Der Besitzer eines biomedizinischen Startups, welches Fortschritte beim therapeutischen Gentransport in Krebs- und Eizellen verzeichnen kann, aber auch mit finanziellen Sorgen zu kämpfen hat, stirbt wegen eines manipulierten Asthmasprays auf einer Wanderung oberhalb von Flims Dorf. Viele kommen als Täter in Frage, von der Ehefrau über Labormitarbeiter, frühere Liebschaften bis zu einem Konkurrenten. Dies bereitet der Kommissarin Laura Crameri der Zürcher Kriminalpolizei einiges an Kopfzerbrechen und Aufklärungsarbeit, bis sie schliesslich die Lösung des Mordfalls findet.

Wie die bisherigen fünf Kriminalromane aus der Feder des emeritierten Genfer Biologieprofessors und mittlerweile renommierten Krimiautors Duri Rungger zeichnet sich auch dieser durch den geschickten Aufbau, die sprachliche Gewandtheit sowie die sorgfältig dargestellten Porträts der darin vorkommenden Personen und die präzise Darstellung einzelner Schauplätze in Zürich, Flims und im deutschen Waldshut aus.

Noch ein abschliessender Tipp: Gerade am Anfang enthält der Krimi Informationen über biomedizinische Grundlagen und molekulargenetische Experimente, die auch für einen alten Genetikprofessor nicht immer leicht nachvollziehbar waren. Sie sind für das Verständnis des Krimis jedoch nicht notwendig! Der interessierte Leser findet im Anhang durch Graphiken didaktisch geschickt ergänzte Darstellungen der erwähnten molekularbiologischen Verfahren.

Prof. em. Dr. med. Hansjakob Müller Medizinische Genetik, Universität Basel

hansjakob.mueller[at]unibas.ch

\section{Teilen Sie Ihre literarischen Entdeckungen mit uns!}

Sie haben ein interessantes Buch gelesen und möchten es einem weiteren Leserkreis vorstellen?

Schicken Sie uns Ihre Buchbesprechung (max. 1200 Zeichen inkl. Leerzeichen) an: redaktion.saez[at]emh.ch
Renata Aebi und Pascal Mösli plädieren dafür, Spiritualität als gesundheitsrelevanten Aspekt zu begreifen. Dazu legen sie jetzt ein Buch vor, das Spiritual Care als interprofessionelle Aufgabe beschreibt.

Die Stärke des Buches liegt im Praxisbezug und richtet sich an alle Professionen des Gesundheitswesens. Die beiden Seelsorgenden aus Sargans und Bern stellen Begegnungen mit Patienten und Behandlungsteams ins Zentrum. So wird der Wert der Spiritualität erfahr- und es wird wahrnehmbar, wie Spiritualität als Ressource fruchtbar gemacht werden kann. Über ein Dutzend Fensterbeiträge ergänzen das Werk. Sie stammen von Fachleuten aus Sozialarbeit, Pflege, Therapie, Psychoonkologie oder Medizin und bringen deren Perspektive ein.

«Spiritual Care funktioniert nur im Zusammenspiel der Berufsgruppen und soll Teil einer gemeinsamen Sorgekultur sein", heisst es. Der Seelsorge, als fachlicher Expertin der Spiritual Care, komme dabei eine koordinierende Funktion zu. «Die beglückenden Erfahrungen von gelingendem Miteinander ermutigen mich, die spirituelle Dimension einer Behandlung in den Blick zu nehmen", erklärt Aebi. «Spiritualität wird durch Wirksamkeitsforschung heute als Teil der Qualität im Gesundheitswesen verstanden", ergänzt Mösli.

Reinhold Meier St. Gallische Psychiatrie-Dienste Süd

Reinhold.Meier[at]psych.ch

\section{Partagez vos découvertes littéraires!}

Vous avez lu un ouvrage intéressant et souhaitez en faire profiter d'autres lecteurs? Envoyez-nous votre critique littéraire (max. 1200 signes, espaces compris) à: redaction.bms[at]emh.ch 